\section{VARIACIÓN DEL PUNTAJE OBTENIDO EN EL EXAMEN NACIONAL DE OBSTETRICIA SEGÚN LICENCIAMIENTO UNIVERSITARIO, 2018}

\section{VARIATION IN THE SCORE OF THE NATIONAL MIDWIFERY EXAMINATION ACCORDING TO UNIVERSITY LICENSURE, 2018}

\author{
Victor Hugo Moquillaza-Alcántara,,a, \\ Grecia Paola Herrera-Chenett ${ }^{2, a}$
}

\begin{abstract}
Sr. Editor. Las universidades a nivel internacional vienen generando cambios con la finalidad de garantizar una buena calidad educativa ${ }^{(1,2)}$. En Perú, la Superintendencia Nacional de Educación Superior Universitaria (SUNEDU) ha establecido estándares, denominados «condiciones básicas de calidad», que deben presentar las universidades para considerarse licenciadas ${ }^{(3)}$; sin embargo, no se ha evidenciado si el licenciamiento podría influir en los resultados en un examen nacional. Por lo cual, el presente estudio tiene como objetivo evaluar la variación de la calificación obtenida en el Examen Nacional de Obstetricia según el estado de licenciamiento universitario, durante el 2018.
\end{abstract}

Estudio transversal donde seevaluóla calificación obtenidapor los participantes del primer Examen Nacional de Obstetricia (ENAOBS), realizado en noviembre del 2018 en Perú, de acuerdo al estado del licenciamiento de la universidad de procedencia. Se incluyeron como participantes a los internos, egresados, bachilleres y titulados en Obstetricia que rindieron el examen.

El cálculo del tamaño de muestra se obtuvo mediante el software libre OpenEpi (http://www.openepi.com). La población total del primer ENAOBS fue de 576 participantes ${ }^{(4)}$ y se consideró una frecuencia hipotética del $50 \%$, un $5 \%$ de límite de confianza y un efecto de diseño de 1; generando una muestra mínima de 231 participantes. Considerando las posibles pérdidas se determinó una muestra final de 250 participantes.

La selección aleatoria se realizó en Excel, tomando como base la lista de participantes del ENAOBS, ordenados

\footnotetext{
Facultad de Salud Pública y Administración, Universidad Peruana Cayetano Heredia. Lima, Perú.

2 Centro Federado de Obstetricia, Universidad Nacional Mayor de San Marcos. Lima, Perú.

a Bachiller en Obstetricia

Recibido: 13/02/2019 Aprobado: 24/02/2019 En línea: 19/03/2019
}

Citar como: Moquillaza-Alcántara VH, Herrera-Chenett GP. Variación del puntaje obtenido en el examen nacional de obstetricia según licenciamiento universitario, 2018. Rev Peru Med Exp Salud Publica. 2019;36(1):145-6.doi: 10.17843/rpmesp.2019.361.4293. alfabéticamente y codificados entre 1 y 576 . Se contactó a los sujetos seleccionados y se les solicitó los datos correspondientes a su usuario y contraseña para poder visualizar sus puntajes; de negarse se procedió a aleatorizar a un nuevo participante.

El estado del licenciamiento fue consultado en la web de la SUNEDU el 24 de enero del 2019, donde las universidades fueron consideradas en base a dos alternativas: licenciadas o en proceso de licenciamiento. Asimismo, la calificación obtenida en el primer ENAOBS fue establecida en un sistema vigesimal, dividido en siete áreas: obstetricia, ginecología, salud sexual y reproductiva, investigación y administración, educación en salud, salud pública y área general (5).

Los datos fueron recopilados en Excel donde se evaluó la calidad de la información, y luego fueron exportados al software STATA versión 14. La variación entre los resultados obtenidos en las universidades licenciadas y en proceso de licenciamiento fue evaluada mediante diferencias de medias (desviación estándar) con la prueba $T$ de Student. Adicionalmente, se calculó la proporción de respuestas correctas, considerando como numerador a la media y como denominador el máximo puntaje posible a obtener.

En todo el proceso se respetó la decisión de los participantes a permanecer en el estudio. Asimismo, toda información identificable fue codificada a fin de garantizar la confidencialidad de los participantes. Los datos obtenidos se encuentran a libre disponibilidad de los lectores: https://doi.org/10.6084/ m9.figshare.7763558.v1

De los 250 participantes del primer ENAOBS, 103 provenían de una universidad licenciada y 147 de una en proceso de licenciamiento. El 96,4\% (236) fueron mujeres y provenían en su mayoría de la Universidad de San Martín de Porres $(13,2 \%)$. Asimismo, el $52,8 \%$ (132) fueron bachilleres y el $21,6 \%$ (54) fueron titulados en Obstetricia. Los participantes provenían, principalmente, de las ciudades de Lima $(21,2 \%)$ y Arequipa $(11,2 \%)$.

En la Tabla 1 se observa que la media de la calificación de quienes provenían de una universidad licenciada fue de 11,8 (DE: 0,7) y de quienes provenían de una universidad en proceso de licenciamiento fue de 11,1 (DE: 0,7) siendo esta diferencia significativa $(p=0,001)$. Quienes provenían de universidades licenciadas obtuvieron una calificación significativamente mayor en las áreas de obstetricia $(p=0.009)$, salud pública $(p=0,002)$ y área general $(p=0,006)$.

Asimismo, el área general e investigación y administración fueron las áreas que presentaron menos acierto, mientras que obstetricia y ginecología fue la de mayor proporción de respuestas correctas. Finalmente, el acierto siempre fue mayor en quienes provenían de una universidad licenciada. 
Tabla 1. Variación de la calificación general y por áreas del Examen Nacional de Obstetricia según el estado del licenciamiento universitario, 2018

\begin{tabular}{|c|c|c|c|c|c|c|c|}
\hline & \multicolumn{6}{|c|}{ Estado del licenciamiento universitario* } & \multirow{3}{*}{ Valor de $p$} \\
\hline & \multicolumn{3}{|c|}{ Licenciada $(n=103)$} & \multicolumn{3}{|c|}{ En proceso $(n=147)$} & \\
\hline & Media & DE & $\%^{\dagger}$ & Media & $\mathrm{DE}$ & $\%^{\dagger}$ & \\
\hline Calificación general & 11,8 & 0,7 & 59,1 & 11,1 & 0,7 & 55,7 & 0,001 \\
\hline \multicolumn{8}{|l|}{ Calificación por áreas } \\
\hline Obstetricia & 4,2 & 0,7 & 69,9 & 3,9 & 0,7 & 65,7 & 0,009 \\
\hline Ginecología & 2,0 & 0,4 & 68,1 & 1,9 & 0,4 & 66,1 & 0,297 \\
\hline Salud Sexual y Reproductiva & 1,5 & 0,4 & 48,9 & 1,4 & 0,4 & 47,7 & 0,462 \\
\hline Investigación y Administración & 0,7 & 0,3 & 43,1 & 0,6 & 0,3 & 40,8 & 0,324 \\
\hline Educación en Salud & 0,7 & 0,3 & 48,8 & 0,6 & 0,2 & 45,0 & 0,123 \\
\hline Salud Pública & 1,9 & 0,4 & 62,3 & 1,7 & 0,4 & 56,7 & 0,002 \\
\hline Área General & 0,9 & 0,3 & 44,3 & 0,8 & 0,3 & 38,8 & 0,006 \\
\hline
\end{tabular}

DE: Desviación estándar

* Según la Superintendencia Nacional de Educación (SUNEDU) al 24 de enero de 2019

† Porcentaje de respuestas correctas, estimado en base a la media / máximo puntaje posible

‡Prueba T de Student

Es preciso indicar que el licenciamiento es un proceso dinámico, por lo que, al momento de realización del estudio, quizás algunas universidades cumplían los criterios de licenciamiento y su denominación como «licenciada» sólo se encontraba en trámite. Aun así, este estudio ratifica la necesidad del licenciamiento universitario a nivel nacional, ya que garantiza condiciones básicas de calidad que brindaría mejores competencias a los estudiantes, lo cual podría traducirse como una mejor preparación académica y de este modo mayores puntajes en exámenes nacionales, permitiéndole así competir por una de las plazas en el Servicio Rural y Urbano Marginal de Salud.

Concluimos que el promedio de calificación del estudiante de Obstetricia proveniente de una universidad licenciada es significativamente mayor en comparación con aquellos estudiantes provenientes de una universidad en proceso de licenciamiento.

Agradecimientos: A Erick Harold Risco Alcántara, por su apoyo en el enrolamiento de los participantes y en la digitación de la información obtenida en la base de datos.

Contribuciones de autoría: MAV y HCGP han participado en la concepción y diseño del artículo, recolección de resultados, redacción del artículo y aprobación de la versión final. Además MAV realizó el análisis e interpretación de los datos.

Financiamiento: El presente trabajo ha sido financiado con un aporte del Fondo Nacional de Desarrollo Científico, Tecnológico y de Innovación Tecnológica (FONDECYT).

Conflictos de interés: Los autores declaramos no presentar conflictos de interés.

\section{REFERENCIAS BIBLIOGRÁFICAS}

1. Campillo A. La universidad en la sociedad global. Isegoría. Revista de Filosofía Moral y Política. 2015;52:15-42. doi: 10.3989/ isegoria.2015.052.01.

2. Altbach PG, Salmi J, editores. El camino hacia la excelencia académica: la constitución de universidades de investigación de rango mundial. 1ra ed [Internet]. Washington: Banco Mundial; 2011. [citado el 26 de enero del 2019] Disponible en: http://www. agenda2035.ec/sites/default/files/El\%20camino\%20hacia\%20 la\%20excelencia\%20universitaria.pdf

3. Superintendencia Nacional de Educación Superior Universitaria [Internet]. Lima: SUNEDU; 2015 [Consultado el 26 de enero 2019]. Condiciones básicas de calidad Disponible en: https:// www.sunedu.gob.pe/condiciones-basicas-de-calidad/

4. Asociación Peruana de Escuelas y Facultades de Obstetricia [Internet]. Lima: ASPEFOBST; 2019. [Citado el 26 de enero del 2019]. Examen Nacional de Obstetricia 2018. Disponible en: https:// www.aspefobst.pe/eventos/examen-nacional-de-obstetricia-2018/

5. Asociación Peruana de Escuelas y Facultades de Obstetricia [Internet]. Lima: ASPEFOBST; 2019 [Citado el 26 de enero del 2019]. Tabla de especificaciones del ENAOBS. Disponible en: https://www.aspefobst.pe/wp-content/uploads/2019/01/Tablade-Especificaciones.pdf

Correspondencia: Victor Hugo Moquillaza Alcántara

Dirección: Av. Honorio Delgado 430, San Martín de Porres. Lima, Perú.

Teléfono: (+051) 982065404

Correo electrónico: victor.moquillaza@upch.pe 